\title{
Gene stacking of multiple traits for high yield of fermentable sugars in plant biomass
}

\author{
Aude Aznar ${ }^{1 \dagger}$, Camille Chalvin ${ }^{1,2 \dagger}$, Patrick M. Shih ${ }^{1}$, Michael Maimann ${ }^{1,3}$, Berit Ebert ${ }^{1,4,5}$, Devon S. Birdseye ${ }^{1}$, \\ Dominique Loqué $e^{1,6,7}$ and Henrik V. Scheller ${ }^{1,7^{*}}$ (B)
}

\begin{abstract}
Background: Second-generation biofuels produced from biomass can help to decrease dependency on fossil fuels, bringing about many economic and environmental benefits. To make biomass more suitable for biorefinery use, we need a better understanding of plant cell wall biosynthesis. Increasing the ratio of C6 to C5 sugars in the cell wall and decreasing the lignin content are two important targets in engineering of plants that are more suitable for downstream processing for second-generation biofuel production.

Results: We have studied the basic mechanisms of cell wall biosynthesis and identified genes involved in biosynthesis of pectic galactan, including the GALS1 galactan synthase and the UDP-galactose/UDP-rhamnose transporter URGT1. We have engineered plants with a more suitable biomass composition by applying these findings, in conjunction with synthetic biology and gene stacking tools. Plants were engineered to have up to fourfold more pectic galactan in stems by overexpressing GALS1, URGT1, and UGE2, a UDP-glucose epimerase. Furthermore, the increased galactan trait was engineered into plants that were already engineered to have low xylan content by restricting xylan biosynthesis to vessels where this polysaccharide is essential. Finally, the high galactan and low xylan traits were stacked with the low lignin trait obtained by expressing the QsuB gene encoding dehydroshikimate dehydratase in lignifying cells.

Conclusion: The results show that approaches to increasing C6 sugar content, decreasing xylan, and reducing lignin content can be combined in an additive manner. Thus, the engineered lines obtained by this trait-stacking approach have substantially improved properties from the perspective of biofuel production, and they do not show any obvious negative growth effects. The approach used in this study can be readily transferred to bioenergy crop plants.
\end{abstract}

Keywords: Plant cell wall, Galactan, Arabidopsis, Pectin, jStack, Xylan, Lignin, Plant engineering

\section{Background}

By taking advantage of the massive energetic potential of plant lignocellulosic biomass, second-generation biofuels can be produced from a broad spectrum of renewable carbon sources without creating direct competition with the food production market. Lignocellulosic biomass is largely composed of secondary plant cell walls, which are thick walls surrounding some differentiated cells such as xylem vessels. Secondary walls are mainly

\footnotetext{
*Correspondence: hscheller@lbl.gov

${ }^{\dagger}$ Aude Aznar and Camille Chalvin contributed equally to this work

${ }^{1}$ Feedstocks Division, Joint BioEnergy Institute, Lawrence Berkeley

National Laboratory, Berkeley, CA 94720, USA

Full list of author information is available at the end of the article
}

composed of cellulose, hemicelluloses, and lignin [1], and constitute the most abundant biomaterials on Earth. Cell wall polysaccharides can be enzymatically degraded to release monosaccharides through a process known as saccharification. Subsequently, microbes can be used to convert monosaccharides to produce bioethanol or other bioproducts. However, several characteristics of lignocellulosic biomass limit the yield of this process, making it expensive [2].

Secondary cell wall polysaccharides are embedded in lignin, a polymer of cross-linked aromatic alcohols that protect the cell wall polysaccharides from enzymatic degradation by plant pathogens and pests, e.g., in case of nematode infection [3], and contribute to biomass 
recalcitrance. Up to $35 \%$ of secondary cell walls are hemicelluloses, which in angiosperms are composed mainly of pentoses such as xylose and arabinose [4]. Because pentoses are less fermentable by microorganisms than hexoses $[5,6]$, increasing the hexose/pentose ratio is one approach to improving biomass for biofuel production. Due to the low density of the lignocellulosic biomass, transportation costs to processing facilities are another limiting factor, especially for biomass from grasses [7]. Engineering of plants with modified secondary cell walls more suitable for downstream processing could reduce costs and facilitate biofuel production from lignocellulosic biomass.

Nevertheless, modifying the cell wall also remains challenging because of its central role in many plant functions. Different groups have already investigated several approaches to making lignocellulosic biomass more suitable for conversion into bioethanol [8]. Genetic engineering efforts to reduce lignin content typically employ techniques to constitutively repress lignin biosynthesis, e.g., by RNAi. However, these strategies frequently result in reduced plant size, and often more than one copy of the biosynthetic gene must be targeted to achieve reduced lignin [9-11]. Recently, we developed a gain-of-function strategy allowing a decrease in lignin content without any visible effect on plant growth [12]. The Arabidopsis thaliana CINNAMATE-4-HYDROXYLASE (C4H) promoter was used to express the Quinate and Shikimate Utilization $B(Q s u B)$ gene encoding the 3-dehydroshikimate dehydratase from Corynebacterium glutamicum in lignified tissues of A. thaliana [12]. By converting 3-dehydroshikimate into protocatechuic acid, the QsuB enzyme produces two effects: (1) it limits the availability of shikimate, a precursor for lignin biosynthesis and a cofactor of hydroxycinnamoyl transferase and (2) it produces an inhibitor of the same transferase [13, 14]. Biomass from plants expressing $Q s u B$ in lignified tissues exhibits a $50 \%$ decrease in lignin content and shows improved saccharification efficiency.

One approach to increase the hexose/pentose ratio in lignocellulosic biomass is to increase the proportion of hexose-rich polysaccharides in secondary cell walls. $\beta-1,4-$ Galactan is entirely composed of galactose residues and is found as sidechains of rhamnogalacturonan $I$ in pectin of primary cell walls [15]. Pectin, including $\beta-1,4-$ galactan, is not abundant in secondary cell walls except in gelatinous fibers. Gelatinous fibers are found in plants such as flax and in tension wood, a specific type of wood that plays a role in maintaining appropriate plant growth under mechanical stress [16]. Tension wood of aspen trees has been reported to contain $10 \%$ of $\beta$-1,4-galactan, which is hypothesized to induce gel-like properties, conferring the contractile driving force of tension wood [16].
Our previous work showed that the glycosyltransferase Galactan Synthase 1 (GALS1) is a $\beta$-1,4-galactan synthase involved in the biosynthesis of pectic galactan in the Golgi apparatus [17]. Constitutive overexpression of GALS1 in Arabidopsis increased the amount of galactose in leaf cell walls by $50 \%$ without an apparent effect on plant growth.

Moreover, the co-overexpression of GALS1 and the cytosolic UDP-glucose/UDP-galactose-4-epimerase 2 (UGE2) [18] under the control of the 35S promoter led to an $80 \%$ increase in galactose in stem cell walls [19]. As UDP-galactose residues are polymerized in the Golgi apparatus, whereas UDP-galactose is synthesized in the cytosol [15], UDP-galactose transport from cytosol to Golgi could become limiting when GALS1 and UGE2 are both overexpressed. Recently, the Golgi-localized UDPRhamnose/UDP-Galactose Transporter 1 (URGT1) was shown to be involved in transport of UDP-galactose into the Golgi apparatus [20]. Overexpression of URGT1 led to increased $\beta$-1,4-galactan accumulation in Arabidopsis leaves, indicating that UDP-galactose transport may indeed be limiting for galactan biosynthesis. In this study, we aimed to overcome the limitation in UDP-galactose in the Golgi lumen by co-overexpressing URGT1 with GALS1 and UGE2.

Another approach to increase the hexose/pentose ratio in lignocellulosic biomass is to decrease the proportion of pentose-rich polysaccharides. The glycosyltransferase Irregular Xylem 7 (IRX7) is involved in the biosynthesis of xylan, a polymer of $\beta$-1,4-linked xylose units, which are highly abundant in secondary cell walls [21]. Compared to wild-type plants, irx 7 loss-of-function mutants have a lower xylan content and exhibit severe dwarfism due to collapsed xylem vessels and the consequent impairment of water and nutrient transport [21]. Using the $V N D 7$ vessel-specific promoter to express the IRX7 coding sequence in the $i r x 7$ mutant, the growth phenotype is rescued while the content of xylose residues in stem cell walls is still reduced by $20 \%$ as compared to non-engineered lines [22]. Here, we utilized this genetically engineered low pentose background and engineered increased galactan content to further increase the C6 to C5 ratio.

The NAC (NAM/ATAF1/CUC2) Secondary cell wall Thickening-promoting factor 1 (NST1) transcription factor is a master regulator controlling secondary cell wall biosynthesis in fiber cells, which provide mechanical support to vessels in stems [23]. NST1 is a potential target for the modification of cell wall thickness. However, its constitutive overexpression leads to the formation of ectopic secondary cell wall thickening and inhibits plant growth [23]. A system allowing overexpression of NST1 without any negative effect on plant growth has been developed 
by Yang et al. [24]. A downstream promoter induced by NST1 is used to express a new chimeric copy of NST1, creating an artificial-positive feedback loop (APFL) and leading to the over-accumulation of NST1 in fiber cells only. Plants expressing a chimeric copy of NST1 under the control of one of its target promoters have fiber cells with thicker secondary cell walls and, accordingly, lignocellulosic biomass obtained from their stems is of a higher density [19, 23].

In the present work, genetic engineering was used to modify cell wall composition and thickness in Arabidopsis, with the aim of increasing the $\mathrm{C} 6$ to $\mathrm{C} 5$ ratio, reducing biomass recalcitrance, and increasing the lignocellulosic biomass density obtained from plant stems for biofuel production.

Overexpression of URGT1 together with GALS1 and $U G E 2$ increased the cell wall galactan content in stems without causing any growth defects. The use of constitutive promoters was compared with the use of secondary cell wall-specific promoters, which limit transgene expression to lignified tissues that constitute the major part of lignocellulosic biomass.

We also stacked several previously described cell wall engineering strategies to evaluate additive effects and compatibility. First, increased galactan content and decreased lignin content traits were combined by overexpressing QSUB together with GALS1, UGE2, and URGT1. Secondly, increased cell wall thickness was combined with higher galactan content and/or lower lignin content through the overexpression of NST1, creating an APFL that increased secondary wall density in interfascicular fiber cells. Third, all these modifications have been combined with a decrease in xylan content by overexpressing the gene sets described above in a vessel-complemented xylan-deficient background [22].

\section{Results}

\section{Design, construction, and stacking of multiple genes} for improved biomass traits

We aimed to overexpress GALS1, UGE2, and URGT1 involved in galactan biosynthesis; the NST1 coding sequence to create an APFL increasing cell wall thickness in fibers; and the QsuB gene responsible for lignin decrease. In this work, the jStack method was used to clone all the transgenes in a single binary vector, allowing a single step transformation of Arabidopsis plants and resulting in the co-segregation of the different transgenes [25]. Five constructs were designed with the combinations of transgenes depicted in Fig. 1.

The constructs named $\mathrm{C} 1$ and $\mathrm{C} 2$ were designed to improve galactan engineering in Arabidopsis (Fig. 1). In $\mathrm{C} 1$, the expression of GALS1, UGE2 and URGT1 coding sequences is under the control of pNOS (Nopaline
Synthase), pMAS (Mannopine Synthase) and pACT2 (Actin 2) constitutive promoters [25], respectively. In $\mathrm{C} 2$, the expression of GALS1, UGE2, and URGT1 coding sequences is under the control of the secondary cell wallspecific $p C 4 H, p I R X 5$, and $p I R X 8$ promoters, respectively. In Arabidopsis, IRREGULAR XYLEM 5 (IRX5), also named $C E S A 4$, encodes one of the catalytic subunits of the cellulose synthase complex [26] and IRREGULAR XYLEM 8 (IRX8), also named GAUT12, encodes a protein with putative galacturonosyltransferase activity that is required for xylan biosynthesis [27].

The construct C3 was designed to increase cell wall thickness specifically in fibers by including the NST1 APFL along with transgenes from the $\mathrm{C} 2$ construct (Fig. 1). The coding sequence of NST1 was fused with the UGE2 coding sequence using a $2 \mathrm{~A}$-peptide as described previously [19]. The 2A-peptide allows coordination of the expression of multiple proteins and polyprotein cleavage in plants [28]. The NST1-2A-UGE2 synthetic sequence is driven by the $p I R X 5$ promoter.

Because we also aimed to reduce the amount of lignin in fibers of the galactan-engineered lines, the QsuB coding sequence [12] was stacked with $\mathrm{C} 2$ construct synthetic genes, resulting in the generation of construct $\mathrm{C} 4$, or with $\mathrm{C} 3$ construct synthetic genes, resulting in the generation of construct $\mathrm{C} 5$ (Fig. 1) In both $\mathrm{C} 4$ and $\mathrm{C} 5$, the expression of the bacterial 3-dehydroshikimate dehydratase encoded by the $Q s u B$ sequence is controlled by the promoter of $C E S A 7$, which is one of the catalytic subunits of cellulose synthase in secondary walls [26].

Wild-type Arabidopsis plants were independently transformed with constructs $\mathrm{C} 1, \mathrm{C} 2, \mathrm{C} 3, \mathrm{C} 4, \mathrm{C} 5$, or the $\mathrm{C} 0$ empty vector control construct. The corresponding T3 generation plants from these lines will be, respectively, named W1, W2, W3, W4, W5, or W0. To further increase the C6:C5 ratio in cell walls, the xylan-engineered line irx7/irx $7 p V N D 7: I R X 7$ was used as genetic background [22]. The irx7/irx7 pVND7:IRX7 line, named here xylan-engineered (XE) line, was independently transformed with constructs $\mathrm{C} 0, \mathrm{C} 4$ or $\mathrm{C} 5$. Plants from these lines were, respectively, named X0, X4, or X5.

\section{Morphological phenotypes of the engineered lines}

In general, no obvious differences were observed in inflorescence stem morphology and growth of W- and $\mathrm{X}$-engineered lines in comparison to Col-0, W0 and X0 controls (Fig. 2), except for some of the W3 and W4 lines, where the inflorescence stems were shorter than those of the controls (Fig. 2c). Interestingly, two of the three X4 lines analyzed were taller at maturity than the X0 line (Fig. 2d). Inflorescence stems of plants from the W5 and X5 lines were not able to stand fully upright (see Additional file 1). Because of the incompatibility of this 


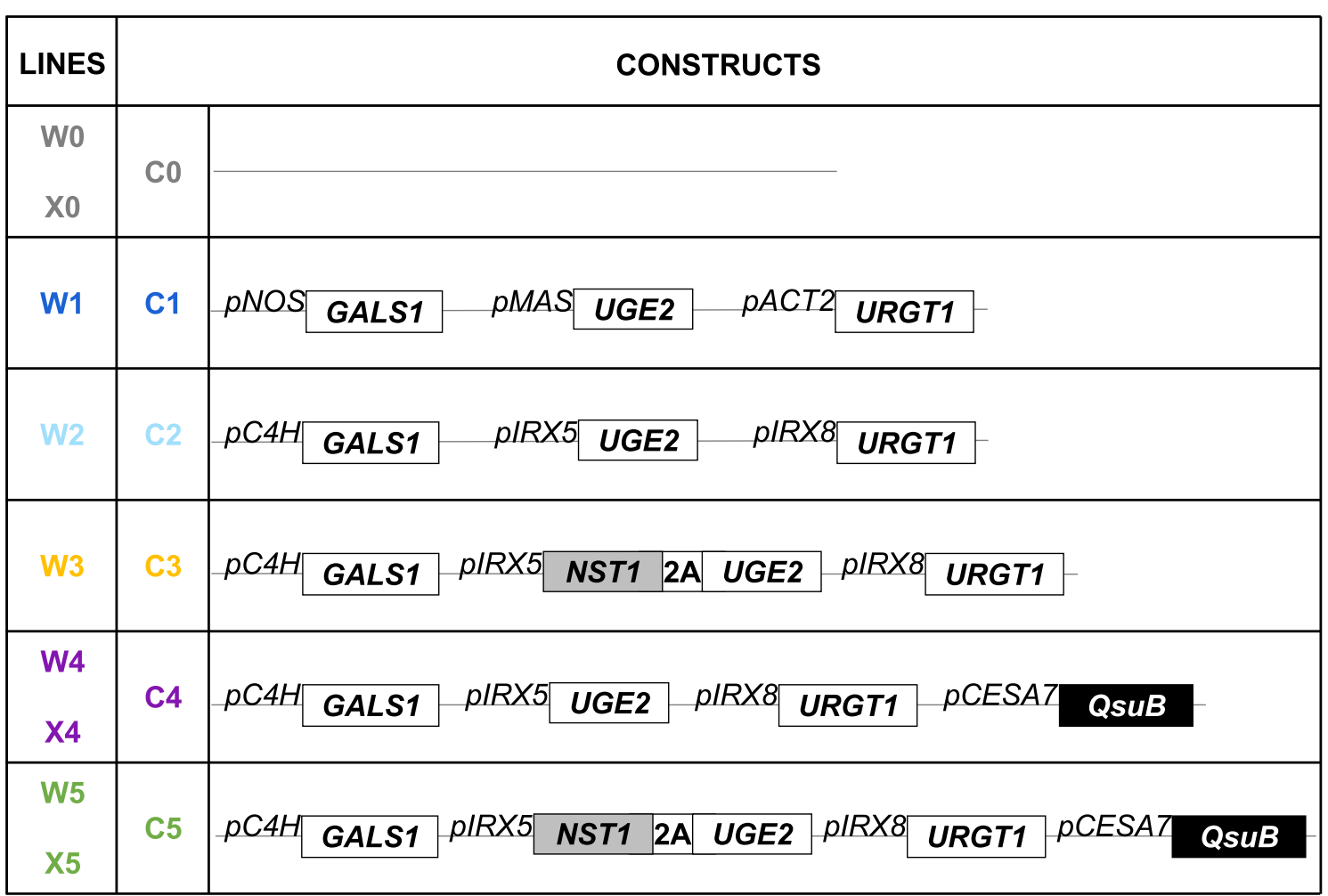

Fig. 1 jStack constructs for the generation of multiple traits engineered Arabidopsis. Lines W0, W1, W2, W3, W4 and W5 express constructs C0, C1 $, C 2, C 3, C 4$ and C5, respectively, in Col-0 WT background. Lines X0 and X4 express constructs C 0 and C4, respectively, in ir 7 //ir 7 pVND7:IRX7 xylan-engineered (XE) background. White squares represent galactan biosynthesis-related ORF, gray square represents secondary cell wall artificialpositive feedback loop-related ORF, and black square represents decreasing lignin-related ORF. pNOS, pMAS and pACT2 are constitutive promoters, and $p C 4 H, p I R X 5, p / R X 8$ and $p C E S A 7$ are promoters specifically expressed in secondary cell wall producing cells. The foot-and-mouth disease virus $2 \mathrm{~A}$ sequence ensures NST1 and UGE2 protein coordinated expression

phenotype with our engineering strategy, W5 and X5 plants were not included in our further studies.

The following analysis focuses on two or three independent engineered lines for each construct: two independent lines for W1 (W1-1, W1-3), W3 (W3-1, W3-5) and three independent lines for W2 (W2-5, W2-4, W2-10), W4 (W4-2 W4-8, W4-1) and X4 (X4-7, X4-10, $\mathrm{X} 4-12)$.

\section{Expression levels of stacked genes in engineered lines}

The accumulation of GALS1, UGE2, URGT1, NST1, and QsuB transcripts was determined by qPCR (quantitative real-time polymerase chain reaction) in inflorescence stem tissues of W- and X-engineered lines (Fig. 3). In general, there was a correlation in expression between the stacked genes in the individual lines, as might be expected since they will be affected. However, independent lines resulting from transformations with the same construct showed large differences in transcript levels of the introduced genes. Compared to controls, transcripts of GALS1 and UGE2 accumulated 43- to 818-fold in
W1 and W2 lines (Fig. 3a). URGT1 transcript levels did not increase substantially in the W1 and W2 lines, but increased three- to 11-fold in several of the W3, W4, and $\mathrm{X} 4$ lines. In the W3, W4 and X4 lines, the transcripts of GALS1 and UGE2 were generally increased even more than in the W1 and W2 lines. In W3-1 and W3-5 lines, NST1 transcripts accumulated, respectively, five- and 42-fold compared to controls. The QsuB transcripts were only detected in stem tissues of W4 and X4 lines, as expected, and levels varied between independent lines (Fig. 3a, b). QsuB transcript accumulations were lower in the W4 and X4 lines than in the QsuB control in which $Q s u B$ is under the control of a stronger $\mathrm{pC} 4 \mathrm{H}$ promoter. In conclusion, healthy T3 plants expressing galactan galactan-engineered genes and NST1 APFL or QsuB were successfully generated for further characterization.

\section{Immunodetection of galactans in stems of engineered lines}

To confirm that expression of genes controlling galactan biosynthesis in fibers resulted in targeted galactan 


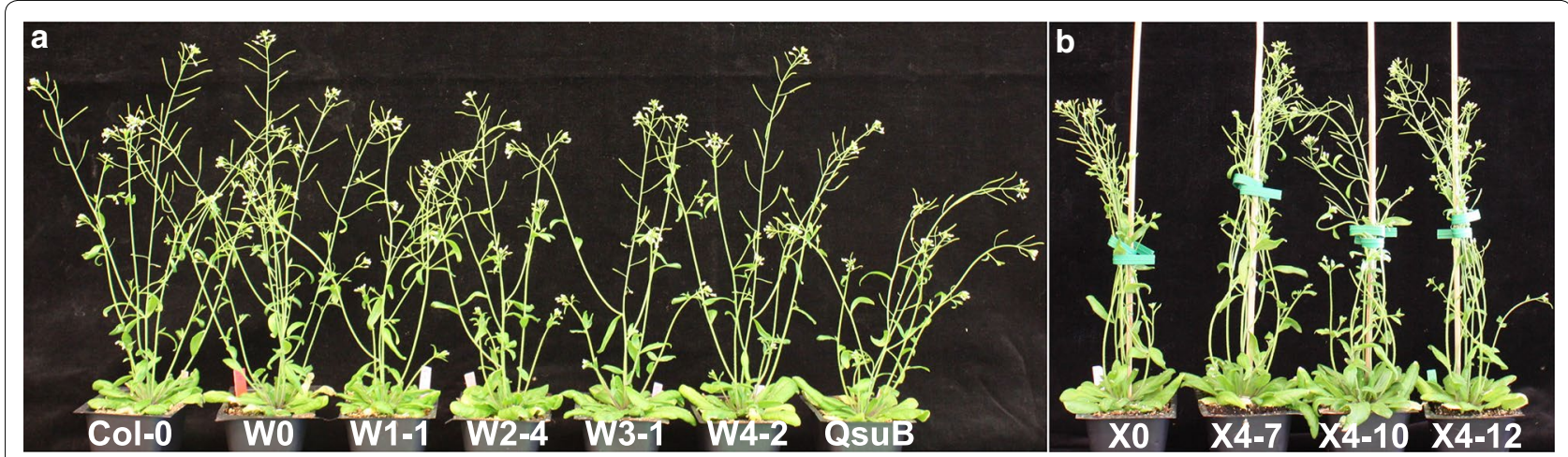

C

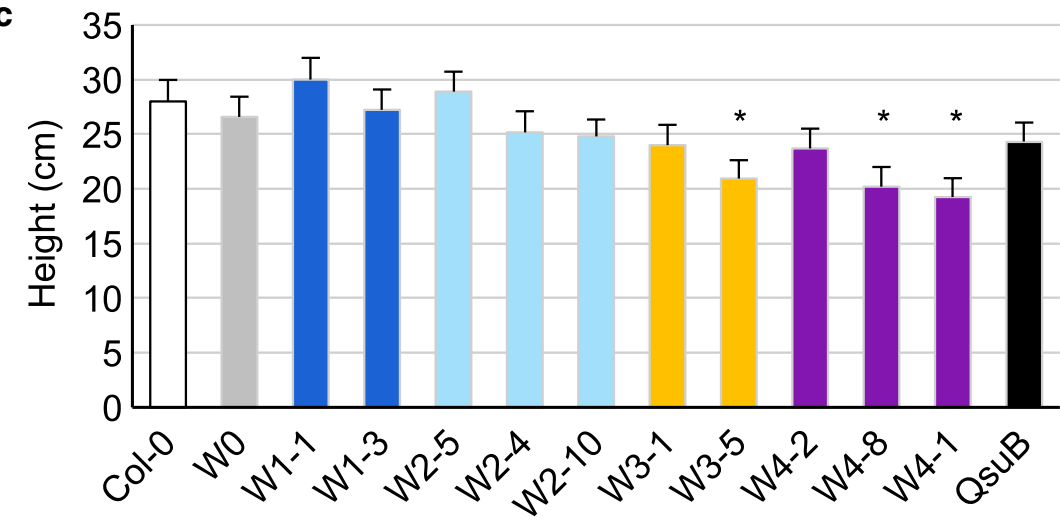

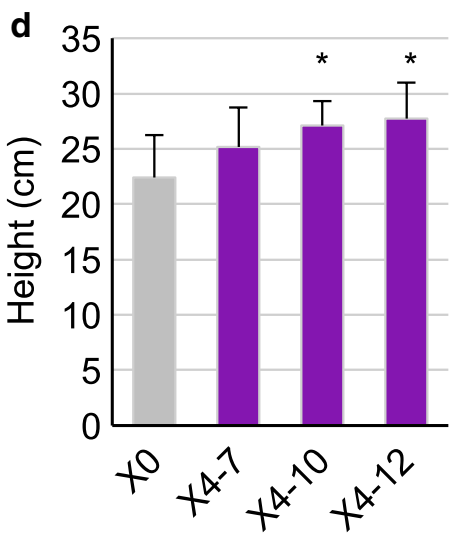

Fig. 2 Morphological phenotypes of engineered lines. Pictures ( $\mathbf{a}, \mathbf{b})$ and main stem height average (c, $\mathbf{d})$ of seven-week-old engineered plants in Col-0 wild type $(\mathbf{a}, \mathbf{c})$ and irx7/irx7 pVND7:IRX7 xylan-engineered (b, d) backgrounds. Bars: SD, $n=6$. ${ }^{*} p<0.05$ with Student's $t$ test, in comparison to WO $(\mathbf{c})$ or XO (d)

enrichment, stem cross-sections from engineered plants were analyzed by immunodetection of galactan using the $\beta$-1,4-galactan-specific LM5 antibody (Fig. 4). While galactan was mainly detected in the cortex, phloem, and pith cell walls of W0 and X0 stems (Fig. 4a, g), it was also present in fiber cell walls of all W- and X-engineered lines. The LM5 signal in fibers was weakly detected in W1-1 and W2-5 stems (Fig. 4b, c). In a W3-1 stem crosssection, no LM5 fluorescence was detected in the pith, but a bright signal emanated from the walls of phloem and fiber cells. In W4-8 stems, LM5 signal was detected in pith, phloem, and fiber cell walls (Fig. 4d, e). While there was no signal in X0 fibers (Fig. 4g), LM5-associated fluorescence was detected in fibers of X4-12 (Fig. 4h).

\section{Cell wall monosaccharide composition of engineered lines}

After TFA (triflouroacetic acid) hydrolysis, the monosaccharide content of dry stems from engineered plant lines was analyzed by High-Performance Anion Exchange Chromatography with Pulsed Amperometric Detection (HPAEC-PAD). Figure 5 highlights galactose and xylose, while Additional file 2 shows all sugar residues measured.
As expected, in the engineered lines in wild-type background, galactose content in the stem was significantly modified compared to Col-0, W0, and pC4H::QsuB controls (Fig. 5a). A 50\% increase in galactose content was detected in W1-8, W2-4, W2-5, W2-10, and W4-2 stems, and up to two- to fourfold increase in W4-8 and W4-1, respectively (Fig. 5a). X4 lines also contained more galactose than $\mathrm{X} 0$ without affecting xylose content to a large extent. X4-7 and X4-10 contained 30\% more galactose and X4-12 contained almost three times more galactose residues than X0 stems (Fig. 5b). In conclusion, when galactan biosynthesis is boosted in fibers, the galactose content is increased in biomass from stems.

\section{Biomass accumulation in inflorescence stems}

To investigate if the large increase in C6 to C5 ratio in the engineered W4-1 line and the X4 lines was associated with a change in overall biomass yield, we determined the mass of senesced stems (Fig. 6). None of the differences were significant (ANOVA, $p>0.3$ ), and this was also true when the X4 lines were analyzed together (ANOVA and Tukey's test, $p>0.1$ for $\mathrm{X} 4$ lines being different from any other lines). 



Fig. 3 Stacked genes expression level in stems of engineered lines. The expression of indicated genes was monitored by qPCR using CDNA from inflorescence stems tissues of W- (a) and X-(b) engineered lines. Gene expression was normalized against the geometric average of transcript levels of three constitutively expressed genes (UBQ10, PP2AA3 and MON1). Bars: SD of the normalized ratio, $n=3$. ND, not detected

Modification of mechanical properties of engineered plant inflorescence stems

To investigate any potential modification in stem mechanical properties, Young's modulus was measured by the three-points bending method to evaluate the inflorescence stem stiffness of the engineered plants. Young's modulus of $\mathrm{pC} 4 \mathrm{H}:: \mathrm{QsuB}$ and $\mathrm{W} 1$ stems were similar to W0 while W2-4, W3-5, W4-8, and W4-1 had a significant reduction in stem stiffness, reflected by a decrease of Young's modulus values (Fig. 7a).

As in W4 engineered lines, X4-10 and X4-12 stem stiffness was significantly reduced by about $30 \%$ compared to 

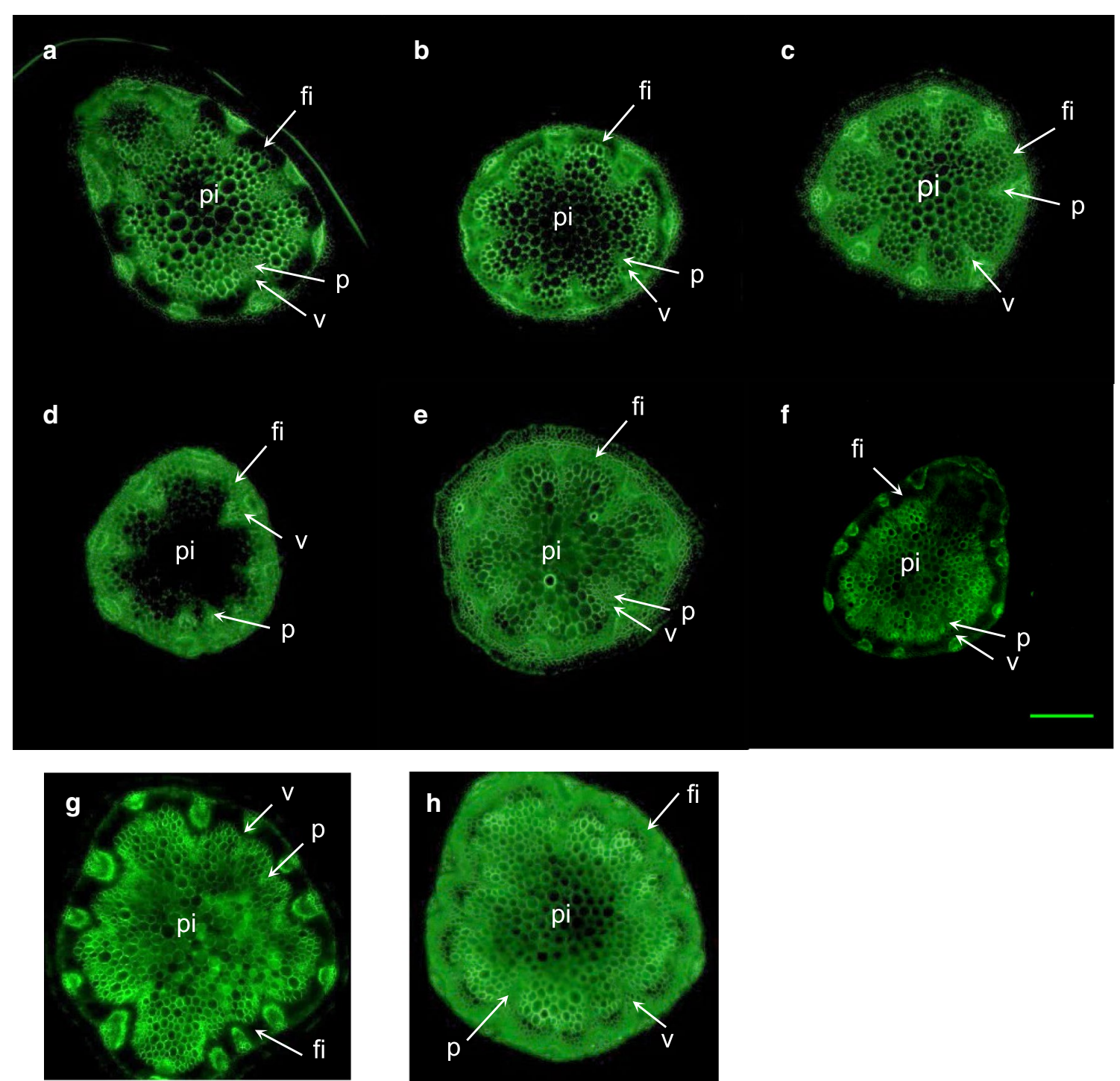

Fig. 4 Galactan detection in stem cross-sections of engineered lines. Cross-section of seven-week-old stem samples were labeled with LM5 (anti- $\beta-1,4-$ galactan) antibody and binding was revealed with an anti-rat IgG antibody coupled with FITC. a W0; b W1-1; c W2-5; d W3-1, e W4-8, f pC4H::QsuB; $\mathbf{g} \times 0, \mathbf{h} \times 4-12$. Scale bar $=20 \mu \mathrm{m}$. The general location of pith (pi), vessels (v), phloem (p), and interfascicular fibers (fi) is indicated

$\mathrm{X} 0$ (Fig. 7b). In conclusion, engineered lines overexpressing the galactan biosynthetic pathway in fiber tissues are affected in stem stiffness independently of the expression of QsuB. However, some lines had only minor reduction in stiffness, and none of the plants showed any morphological differences when compared to the control lines.

\section{Saccharification efficiency of engineered lines}

Saccharification experiments were performed on engineered biomass samples pretreated with hot water at $120{ }^{\circ} \mathrm{C}$, using the Cellic CTec 2 commercial cellulase enzyme blend alone or in combination with an endo- $\beta-1,4-$ galactanase. Saccharification efficiency was evaluated by measuring the amount of reducing sugars (pentoses and hexoses) released at different time points using a colorimetric DNS assay. Compared to W0 and Col-0 controls, biomass from W1, W2, and W3 lines released a similar amount of fermentable sugars, ranging between 260 and $290 \mu \mathrm{g}$ Glc eqv/mg of dry biomass or slightly less in the case of the W3-1 line (Fig. 8). However, the W4 lines (W4-1, W4-2, and W4-8) released 30 to $54 \%$ more reducing sugars when hydrolyzed by cellulases. Compared to Cellic CTec2 hydrolyzed W0 stem biomass, QsuB and W4-8 stem biomass treated with the same enzymatic cocktail released 35 and $48 \%$ more reduced sugars, respectively. In both W- or X-genetic 

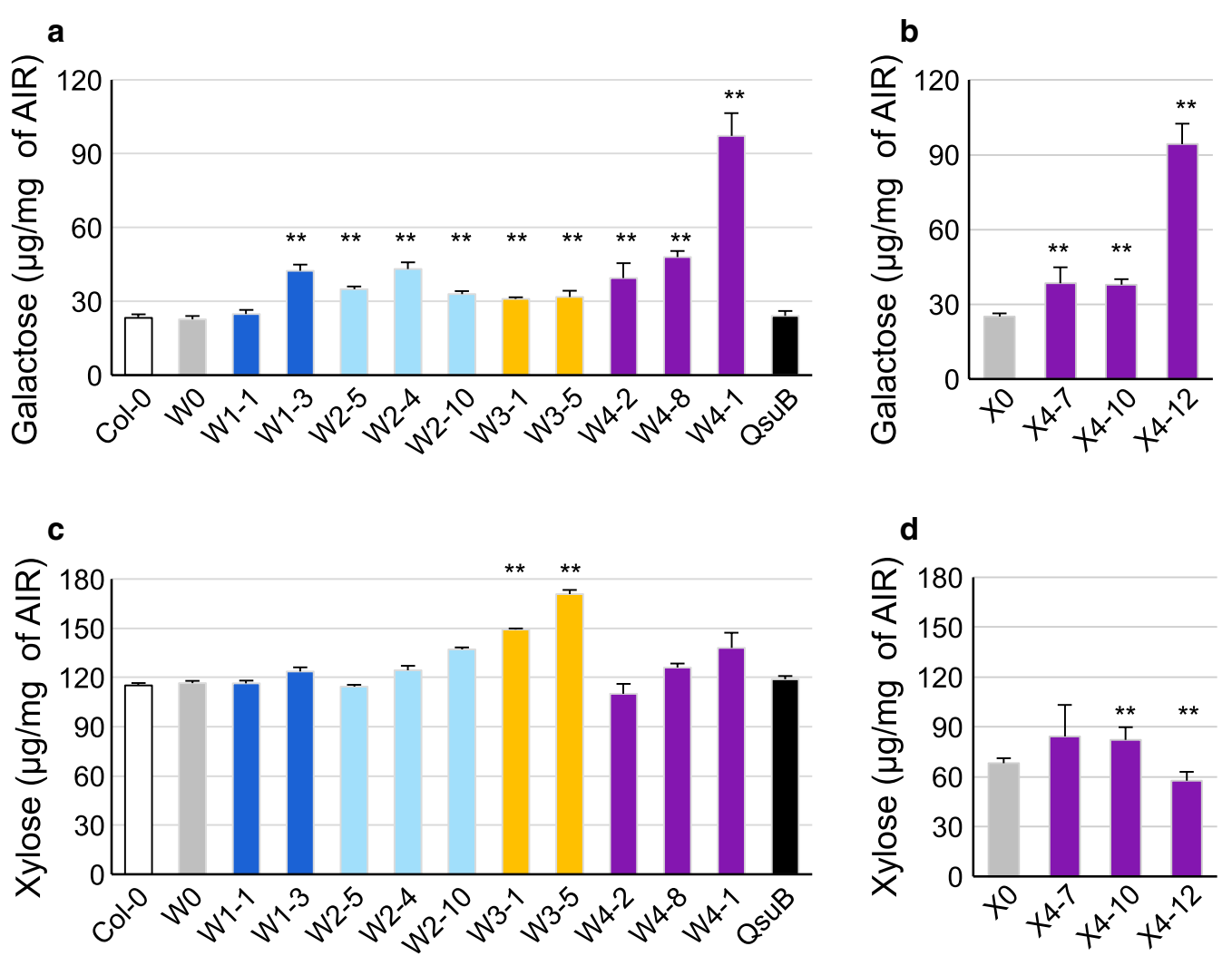

Fig. 5 Galactose and xylose content in stem cell walls of engineered lines. Galactose $(\mathbf{a}, \mathbf{b})$ and xylose $(\mathbf{c}, \mathbf{d})$ content from alcohol insoluble residue (AIR) of stem cell walls of W- $(\mathbf{a}, \mathbf{c})$ and X-(b, d) engineered lines, hydrolyzed with TFA acid, and analyzed by HPAEC-PAD. Sugar residues not show here are displayed in Additional file 2. Error bars: $\mathrm{SD}, n=3 .{ }^{* *} p<0.01$ with Student's $t$ test, in comparison to W0 (a, c) or X0 (b, d)

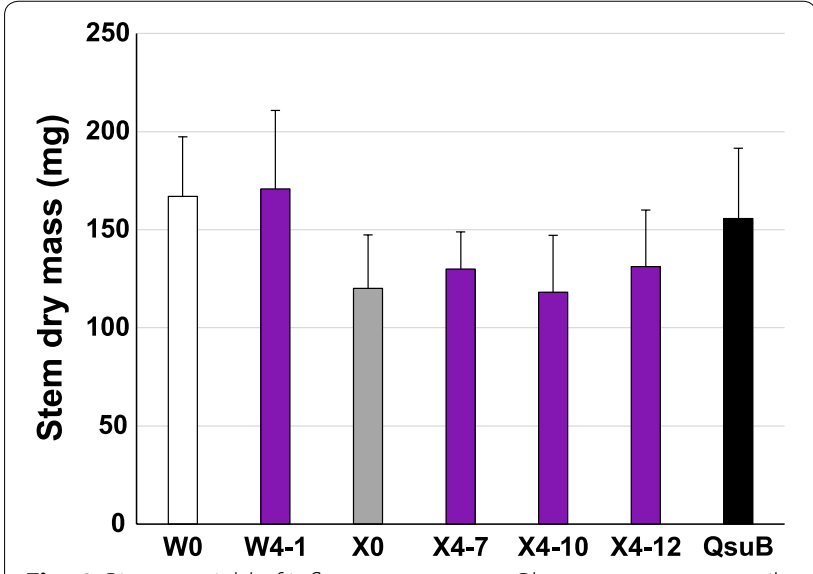

Fig. $\mathbf{6}$ Biomass yield of inflorescence stems. Plants were grown until maturity and the mass of the senesced, dry stems determined. The data are averages $\pm S E$ for four separate experiments, each with three plants per genotype. No significant differences were found (ANOVA, $p>0.3)$

backgrounds, very high saccharification efficiencies were obtained with biomass from plants carrying the construct C4. In X4 plants, the efficiency of both hot water pretreatment and saccharification was highly increased. Additional treatment with an endo- $\beta$-1,4-galactanase had a significant effect (ANOVA, $p<0.01$ ) and on the average released $9 \%$ additional sugars. In the W4 lines, 73\% more sugar was released with the combination of Ctec2 and galactanase as compared to the control plants.

\section{Discussion}

Overexpression of GALS1, UGE2, URGT1, and NST1 and additional expression of $Q s u B$ in W- and X-Arabidopsis genetic backgrounds

In this report, multiple traits beneficial to biofuel production were combined in Arabidopsis using the jStack cloning method. Up to three individual traits were simultaneously introduced via a gene stacking approaches into wild-type (W-) and xylan-engineered (X-) backgrounds (Figs. 1,2). Plants were generated to overexpress three key genes (GALS1, UGE2, and URGT1) involved in galactan biosynthesis, and showed increased expression under both constitutive and fiber-specific promoters in T3 generation (Fig. 3). These W1 and W2 lines did not show a morphological phenotype compared to Col0 , and exhibited similar development during their life 
a

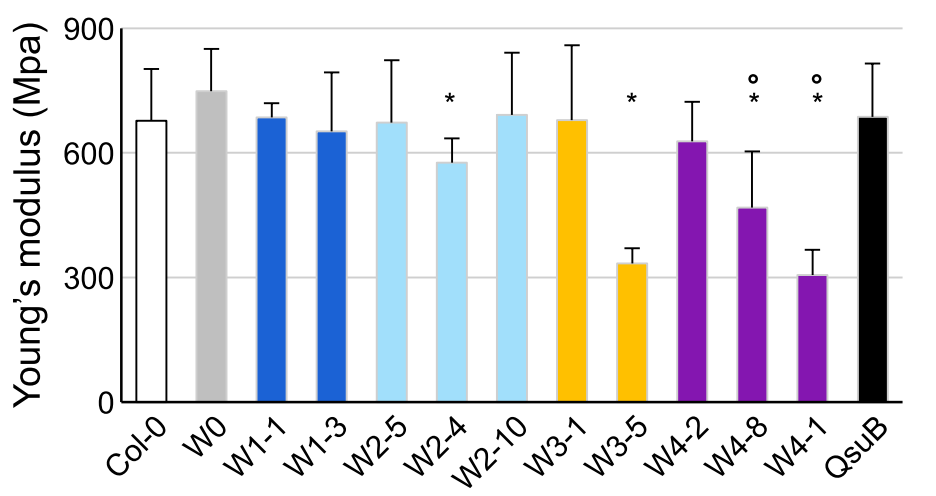

b

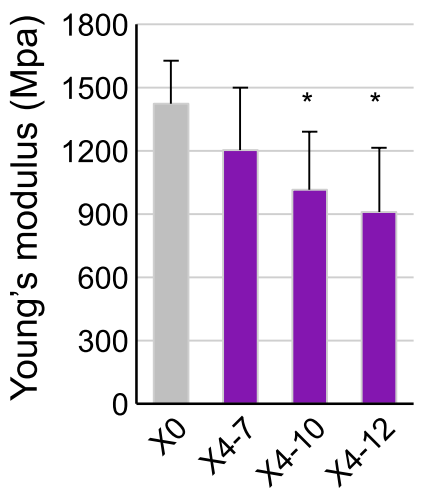

Fig. 7 Young's modulus measurements of inflorescence stems. The three-point bending force of the main inflorescence stem of seven-week-old W- (a) or X-(b) engineered lines was tested. Error bars: SD, $n=6$. $^{*} p<0.01$ with Student's $t$ test, in comparison to W0 (a) or X0 (b). ${ }^{\circ} p<0.05$ with Student's $t$ test, in comparison to QsuB

cycles. Lines expressing the C4 construct displayed opposite phenotypes in the $\mathrm{W}$ - and in $\mathrm{X}$-genetic backgrounds (Fig. 2). While the inflorescence stems of engineered lines tended to be shorter in the W-background, they had the same height or were taller in the X-genetic background.

Lines in the W- or X-genetic backgrounds transformed with all the traits in combination (construct $\mathrm{C} 5$ ) show poor growth and weak stems. Thus, this may be indicative that brute-force overexpression strategies may have been exhausted and, in this specific combination, it is quite possible that we have reached the limits of genetic engineering. Alternatively, using different promoters or expressing the NST1 APFL independently from UGE2 could show different outcomes, as the increased expression of NST1 results in higher than optimal level of expression of $Q s u B$ when the promoter ( $p C e s A 7)$ used to drive its expression is induced by the NST1 transcription factor. Testing different promoter combinations could likely overcome the detrimental phenotype.

Using the jStack cloning method, we generated engineered lines carrying all the transgenes at the same locus and the use of a tissue-specific promoter allowed the coordinating spatio-temporal expression of the desired transgenes. Because we had experience with a limited number of strong fiber-specific promoters, we decided to use the 2A peptide to stack genes in addition to the jStack approach. This technique has previously been used successfully by others and in our laboratory. However, in W3-1 and W3-5 stem sections, the presence of the APFL driven by NST1 did not appear to result in a substantial increase of cell wall thickness in fibers compared to what we had observed in previous studies [19, 24]. As mentioned before, a better comprehension of the network regulation of cell wall-related genes and a different sequence arrangement could possibly lead to the desired increase in fiber cell wall density. Alternatively, it is possible that the enhancement of galactan biosynthesis in fibers with promoters that are responsive to NST1 may cause a drastic substrate competition for UDP-glucose between the cellulose and galactan biosynthesis pathways. That could perhaps lead to reduced ability to also increase cellulose and hemicellulose biosynthesis by overexpression of NST1.

\section{Engineered plants showed an increase of galactan in fibers} Previously, we showed that the overexpression of UGE2 and GALS1 driven by $35 \mathrm{~S}$ promoter in Col-0 plant leads to $80 \%$ more galactose in the cell wall of stems [19]. Here, we engineered plants able to accumulate up to $150 \%$ more galactose in stem cell walls compared to the wild type. However, our qPCR data did not confirm increased URGT1 expression in all of our engineered lines relative to the control plants (Fig. 3a, b). Some lines did show a three- to 11-fold increase in URGT1 expression (i.e., W4-1 and X4-12) and these showed the highest increase in cell wall galactose accumulation, indicating the importance of boosting the expression of this gene. Expressing URGT1 under a stronger fiber-specific promoter or inserting its native introns could perhaps result in producing even more galactan in cell walls due to increased transport of UDP-galactose into the Golgi lumen. LM5 immunodetection showed that the additional galactose is located in fibers and assembled into galactan polymers (Fig. 4). Moreover, by introducing more galactan in fibers of $\mathrm{XE}$, we demonstrated that it is conceivable to produce dicot plants designed to have reduced xylan content and a high amount of C6 sugars in the secondary cell wall of fibers (Figs. 4, 5, Additional file 2). The C6/C5 sugar ratio 

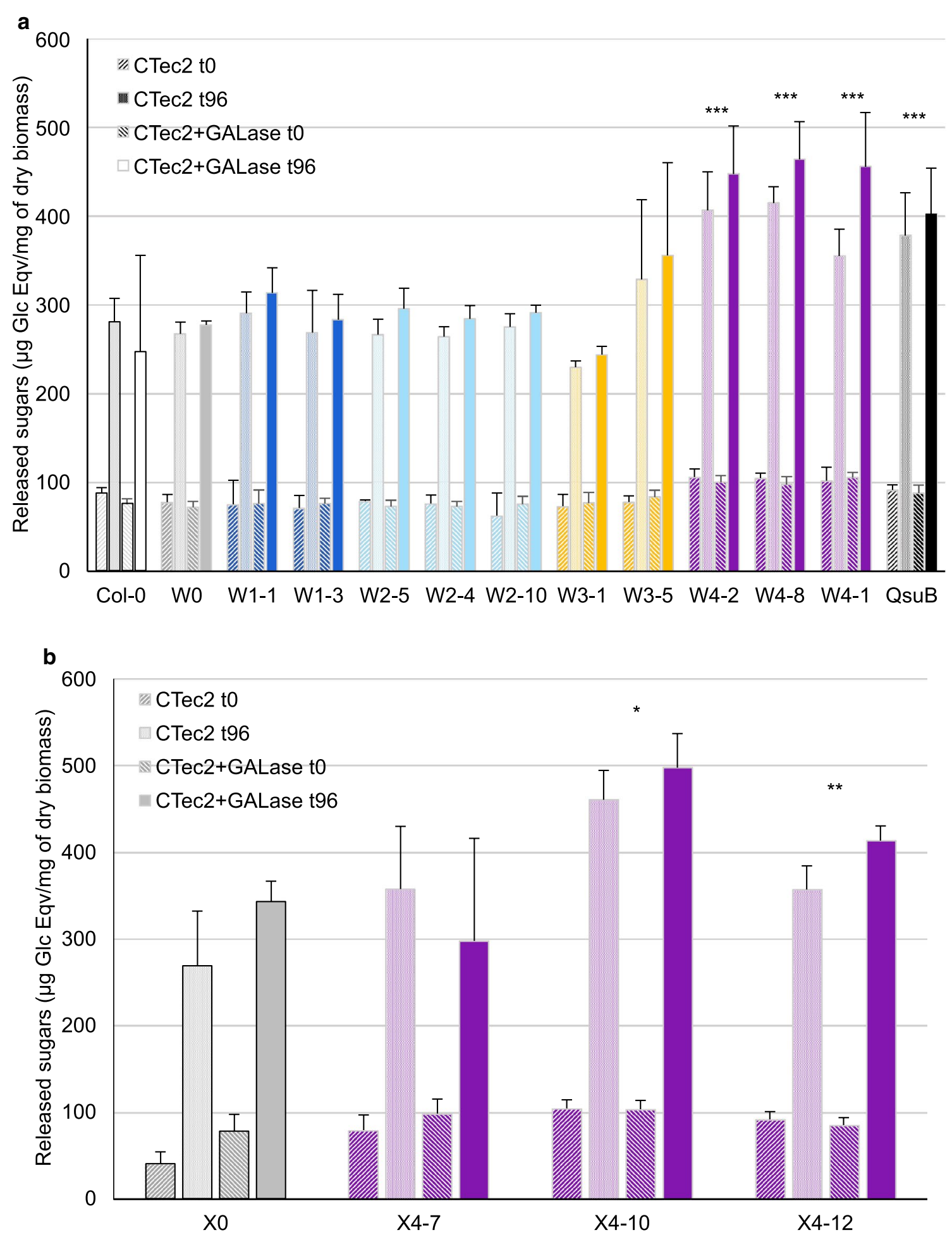

Fig. 8 Saccharification assays of multiple traits engineered lines. Biomass from dry stems of $W$ - $(\mathbf{a})$ and $X$-(b) engineered lines after hot water pretreatment was assessed for saccharification efficiency with CTec2 enzyme cocktail alone or CTec2 complemented with endo- $\beta$-1,4-galactanase at 0 and $96 \mathrm{~h}$. The amount of released sugars was tested with a DNS assay. Bars: SD, $n=3$. The sugar release following enzyme treatment was analyzed by 2-factor ANOVA, followed by Dunnett's multiple comparison test. ${ }^{*} p<0.05,{ }^{* *} p<0.01,{ }^{* * *} p<0.001$ in comparison to W0 (a) or X0 (b). The effect of adding galactanase was significant across all the lines (ANOVA, $p<0.01$ ) but the interaction between galactanase and genotype was not significant 
determined by TFA hydrolysis of cell wall preparations of the $\mathrm{X} 4-12$ plant line is 2.66 as compared to 0.80 in Col0 , corresponding to a 3.3-fold increase (Additional file 2). Assuming that TFA hydrolysis-resistant cellulose levels are the same in all the plants, we estimated a $\mathrm{C6} / \mathrm{C} 5$ ratio of 6.3 in the best X4 line versus 2.6 in Col-0, corresponding to a 2.4-fold increase.

We also demonstrated that the low lignin trait conferred by QsuB engineering is compatible with our hexose-enrichment strategies. Indeed, QsuB expression does not interfere with the accumulation of galactose (Fig. 5) in contrast to the biomass densification trait controlled by APFL. In lines W4-8 and W4-1, QsuB and galactan biosynthesis gene expression seems to have a synergistic effect (Fig. 5a).

\section{Galactan content in fibers can impact stem stiffness}

Modifying the composition of cell walls in stem fibers could affect the mechanical properties of the entire organ. Such modifications could be advantageous to prevent lodging or, on the contrary, enhance lodging susceptibility. To address this question beyond the macroscopic phenotype, we tested stem stiffness with a three-point bending test. Our results showed no substantial effect on stem stiffness in most of the lines (Fig. 7). However, some lines carrying the $\mathrm{C} 4$ construct, in both the Wand X-genetic background, showed a decrease in stiffness, which was not observed in the QsuB control line. This decrease in stiffness was observed in the same lines where the elevated contents of galactose were found (Figs. 5, 7). In these plants, we may have reached the critical point where accumulation of galactan impacts tissue properties and consequently stem mechanical properties. Indeed, galactose content in the cell wall has been demonstrated to impact mechanical properties of Arabidopsis leaves [29]. However, we cannot conclude from the data whether the decreased stiffness in the W4 and X4 lines is due only to the high galactan content or due to the combination of high galactan with low lignin mediated by $Q s u B$ expression.

\section{Optimized C6/C5 sugar ratios are compatible with saccharification improvement traits}

Because most microbes used for conversion into biofuels and bioproducts are more efficient in metabolizing C6 than C5 sugars, we aimed to increase the C6/C5 sugar ratio to optimize plant biomass for biofuels production.

The saccharification assays conducted in this study showed that our engineered plants released more C6 sugars in both the wild-type and in a low C5 sugar (XE) background (Fig. 8). The best engineered line resulted from fiber-specific overexpression of galactan biosynthesis-related genes and the bacterial gene QsuB. Previously, we have shown that the expression of $Q s u B$ itself doubled the saccharification efficiency of Arabidopsis biomass [12]. QsuB expression leads to a decrease of G/S ratio and an increase of $\mathrm{H}$-units in lignin, and biomass of QsuB-expressing plants is more easily hydrolyzed by the Cellic CTec2 enzymatic cocktail than control biomass [12]. In our experiments, the same phenomenon was observed with the QsuB control line. In W4 and X4 lines, the improvement in saccharification due to QsuB combined with the galactan increase in fibers could have an additive effect, resulting in an even better sugar release.

\section{Conclusion}

Here, we have engineered plants with up to a 3.3-fold increase of the C6/C5 sugar ratio in the TFA-hydrolyzable biomass fraction and with reduced lignin. No morphological differences were observed in these plants, except for a slight decrease in stem stiffness and change in height in some of the lines. No significant changes in stem biomass accumulation were observed. The approach demonstrated here can be transferred to bioenergy crops such as poplar (Populus sp.) and possibly also to bioenergy grasses in which galactan is not an abundant polymer. In the absence of mutants in xylan biosynthesis, an alternative method to obtain a similar reduction in xylan specifically in fibers could be achieved by targeted CRISPR/Cas 9 mutagenesis directed against a xylan biosynthesis gene in fiber cells [30]. The effect of changing the biomass in bioenergy crops on agronomical performance is a key question to be resolved and ultimately will require field tests. The galactan-engineered lines described in this study are a valuable tool for further investigation of the potential relationship between galactan content in the secondary cell wall, mechanical properties, and stress responses.

\section{Methods \\ jStack cloning}

The jSTACK DNA assembly method was used as previously described to assemble the five binary plasmids designed for plant cell wall engineering (Fig. 1) [25]. Level 0, level 1, and level 2 assemblies are further detailed in Additional file 4. The binary vector pYB3301 conferring Basta $^{\circledR}$ (glufosinate) resistance to transformed plants was used for all gene stacks [25]. To ensure that all gene cassettes were correctly assembled, junctions were sequenced using the SimplySeq DNA Sequencing service provided by Quintara Biosciences (South San Francisco, $\mathrm{CA}$ ). All sequences and plasmids developed in this project are further described in Additional files 3 and 4 and will be made publicly available through the Inventory of Composable Elements (ICE) repository [31]. 


\section{Plant lines and growth conditions}

All Arabidopsis (Arabidopsis thaliana (L.) Heyhn.) wildtype and mutant plant lines used in this study are in the Columbia (Col-0) background. $p C 4 H:: Q s u B$ and irx7/ irx 7 VND7:IRX7 lines have been described before [12, 22] and were obtained from the Joint BioEnergy Institute Registry (http://www.acs-registry.jbei.org). After being stratified at $4{ }^{\circ} \mathrm{C}$ for 4 days, seeds were grown in soil at $22{ }^{\circ} \mathrm{C}$ in a 10 -h photoperiod for 4 weeks and then moved to a 16-h photoperiod. Agrobacterium tumefaciens strain GV3101 was transformed with the generated plasmids by electroporation [32] and used for plant transformation by floral dip [33]. Seeds from the transformed plants were harvested, sterilized in $10 \%$ bleach in ethanol for $10 \mathrm{~min}$, and then grown on plates containing MS media $(0.5 \times$ Murashige and Skoog salts, $7 \mathrm{~g} / \mathrm{l}$ agar, $10 \mathrm{~g} / \mathrm{l}$ sucrose) with $25 \mu \mathrm{g} / \mathrm{ml}$ glufosinate ammonium for selection and stratified for 4 days at $4{ }^{\circ} \mathrm{C}$. Plates were then transferred to a growth chamber at $22{ }^{\circ} \mathrm{C}$ with 10 -h photoperiod for 7-10 days. Glufosinate-resistant seedlings were transferred to soil and grown for 4 weeks at $22{ }^{\circ} \mathrm{C}$ in 10 -h photoperiod and then transferred to a $16-\mathrm{h}$ photoperiod.

\section{Transgene expression analysis by qPCR}

Samples of seven-week-old main inflorescence stems were ground in liquid nitrogen and RNA was extracted using TRIzol reagent (Thermo Fisher). RNA was treated with DNase I (AMPD1-1KT, Sigma-Aldrich) to eliminate DNA contaminants and cDNA was synthesized using iScript Reverse Transcription Supermix (Bio-Rad, 1708840 ) with $750 \mathrm{ng}$ RNA as a template in a $10 \mu \mathrm{l}$ reaction volume. The expression level of GALS1, UGE2, URGT1, NST1, and QsuB was measured by quantitative PCR using the StepOnePlus Real-Time PCR system (Applied Biosystems) according to the conditions described in Czechowski et al. [34] using StepOne 2.0 software (Applied Biosystems). Primers were designed to amplify cDNAs from both endogenous and transgenic mRNAs of GALS1, UGE2, URGT1, and NST1. The expression level measured for these genes is a combination of the expression levels of the endogenous copy and the transgenic copy. Gene expression was analyzed using the $\Delta \Delta C_{T}$ method [35] and normalization against the geometric mean of the transcript levels of three reference genes [36]. The constitutively expressed reference genes used (UBQ10 (At4g05320), PP2AA3 (At1g13320), MON1 (At2g28390)) have been validated by Czechowski et al. [34]. Primer sequences are available in Additional file 3.

\section{Immunofluorescence microscopy}

The top and base $2.5 \mathrm{~cm}$ of main stems from seven-weekold plants (three stems/line) were harvested and fixed overnight at $4{ }^{\circ} \mathrm{C}$ in fixative solution (4\% paraformaldehyde in $50 \mathrm{mM}$ piperazine-N-N'-bis(2-ethanesulphonic acid), 5 mM EGTA, pH 6.9). Fixed stem sections were embedded in $7 \%$ agarose and $100 \mu \mathrm{m}$ thick sections were generated using a Leica VT1000S vibratome. Stem sections were labeled with monoclonal antibody LM5 (PlantProbes, Leeds, UK), which recognizes 1,4-linked $\beta$-galactan $[37,38]$. Stem sections were washed three times with phosphate-buffered saline (PBS) and incubated for $1.5 \mathrm{~h}$ at room temperature with LM5 or LM10 antibody diluted tenfold in PBS with $5 \%$ milk protein. After three washes in PBS, stem sections were incubated for $1.5 \mathrm{~h}$ in the dark at room temperature with anti-rat IgG secondary antibody coupled with FITC (Fluorescein IsoThioCyanate) diluted 100 -fold in PBS with $5 \%$ milk protein. Stem sections were finally washed three times with PBS and stored overnight at $4{ }^{\circ} \mathrm{C}$ in the dark. Immunostained stem cuts were mounted on slides in a glycerol anti-fade solution (CitiFluor AF1, Agar Scientific) and pictures were taken using a DM6 B epifluorescence microscope (Leica) under blue light (L5 filter, Leica). Images were acquired with a C11440 Hamamatsu camera monitored by the LAS X software (Leica) and then analyzed with ImageJ [39].

\section{Monosaccharide composition analysis}

Whole inflorescence stems from dry mature plants were ground in vials with five metal beads using a Retch mill, and alcohol-insoluble residue (AIR) was prepared and enzymatically destarched as described by Harholt et al. [40]. AIR samples $(1 \mathrm{mg})$ were subsequently hydrolyzed with $2 \mathrm{M}$ trifluoroacetic acid (TFA) for $1 \mathrm{~h}$ at $120^{\circ} \mathrm{C}$. TFA was removed under vacuum overnight and hydrolysis products suspended in $1 \mathrm{ml}$ water at $30{ }^{\circ} \mathrm{C}$ for $30 \mathrm{~min}$. The monosaccharide composition was determined in 15-fold diluted hydrolysates by high-performance anion exchange chromatography coupled with a pulsed amperometric detection (HPAEC-PAD) using an ICS-5000 ion chromatography system with a CarboPac PA20 column (Dionex Thermo Scientific) as described [41, 42].

\section{Young's modulus measurement}

Sections of main inflorescence stems from 25 to $75 \mathrm{~mm}$ above the rosette were taken from seven-week-old plants and three-point flexural tests were performed using a 4500 series Instron universal testing machine (series IX automated materials testing system, http://www.instron.co.uk). Flexural three-point bending stiffness (Young's modulus) was calculated according to the standard equations [43].

\section{Analysis of biomass saccharification efficiency}

Saccharification efficiency was analyzed after hot water pretreatment of ground biomass from senesced stems. 
Samples $(10 \mathrm{mg})$ were mixed with $340 \mu \mathrm{l}$ of water and incubated for $30 \mathrm{~min}$ at $30{ }^{\circ} \mathrm{C}$ with shaking, autoclaved for $1 \mathrm{~h}$ at $120{ }^{\circ} \mathrm{C}$, and cooled down to room temperature. Enzymatic saccharification was initiated by adding $650 \mu \mathrm{l}$ of saccharification mix ( $75 \mathrm{mM}$ citrate buffer $\mathrm{pH} 5$; $115 \mu \mathrm{g} / \mathrm{ml}$ tetracycline; $1 \% \mathrm{v} / \mathrm{w}$ Cellic CTec2 enzyme mix (Novozymes)) to pretreated samples. In some samples, endo-1,4- $\beta$-galactanase from Aspergillus niger (Megazyme) was included at a concentration of $52 \mathrm{U} / \mathrm{mg}$ of biomass. The reaction was carried out at $50{ }^{\circ} \mathrm{C}$ for $96 \mathrm{~h}$ with shaking. Reducing sugar concentration was measured at $t=0 \mathrm{~h}, t=20 \mathrm{~h}, t=48 \mathrm{~h}, t=72 \mathrm{~h}$ and $t=96 \mathrm{~h}$ using the colorimetric dinitrosalicylic acid (DNS) assay [44]. Samples were centrifuged for $3 \mathrm{~min}$ at $13,000 \mathrm{xg}$ to pellet biomass and a $10 \mu \mathrm{l}$ aliquot of supernatant was diluted in $20 \mu \mathrm{l}$ of $100 \mathrm{mM}$ citrate buffer $\mathrm{pH}$ 5. Ninety microlitre of DNS reagent $(0.4 \mathrm{M} \mathrm{NaOH} ; 300 \mathrm{~g} / \mathrm{l} \mathrm{KNa}$ tartrate; $10 \mathrm{~g} / \mathrm{l} \mathrm{DNS}$ ) was added and the mix was heated at $90{ }^{\circ} \mathrm{C}$ for $10 \mathrm{~min}$. The absorbance was measured at $540 \mathrm{~nm}$ and reducing sugar concentration was quantified (in glucose equivalents) using glucose solutions as standards.

\section{Statistical analysis}

Statistical analysis was done with Students $t$ test or with ANOVA as indicated in the figure legends. ANOVA and multiple comparisons (by Tukey's and Dunnett's tests) were done using XLSTAT (Addinsoft, New York).

\section{Sequence IDs}

The promoters and coding sequences used in the gene constructs relate to the following IDs: AtIrx5, At5g44030; AtIrx8, At5g54690; CesA7, At5g17420; GalS1, At2g33570; UGE2, At4g23920; URGT1, At1g76670; NST1, At2g46770; UBQ10, At4g05320; PP2AA3, At1g13320; MON1, At2g28390; QsuB, YP_001137362.1. For further details, see Additional file 3.

\section{Additional files}

Additional file 1. Phenotypes of W5 and X5 engineered lines.

Additional file 2. Monosaccharide composition of stem cell walls of engineered lines. Alcohol-insoluble residue (AIR) of stem cell walls of W(A) and X- (B) engineered lines, hydrolyzed with trifluoroacetic acid, and analyzed by HPAEC-PAD. Bars: SD, $n=3$.

Additional file 3. Primers used for jStack cloning and qPCR transcript level monitoring.

Additional file 4. Summary of jStack constructs.

\section{Authors' contributions}

DL and HVS conceived and supervised the study. AA, CC, PMS, and MM planned and conducted experiments and analyzed data. BE and DB assisted with experiments and data analysis. AA, CC and HVS wrote the manuscript. All authors read and approved the final manuscript.

\section{Author details}

${ }^{1}$ Feedstocks Division, Joint BioEnergy Institute, Lawrence Berkeley National Laboratory, Berkeley, CA 94720, USA. ${ }^{2}$ Ecole Normale Supérieure de Cachan, 94230 Cachan, France. ${ }^{3}$ Technical University of Denmark, 2800 Kgs. Lyngby, Denmark. ${ }^{4}$ Department of Plant and Environmental Sciences, University of Copenhagen, 1871 Frederiksberg, Denmark. ${ }^{5}$ School of BioSciences, University of Melbourne, Melbourne, VIC 3010, Australia. ${ }^{6}$ INSA de Lyon, CNRS, UMR5240, Microbiologie, Adaptation et Pathogénie, Université Claude Bernard Lyon 1, 69622 Villeurbanne, France. ${ }^{7}$ Department of Plant and Microbial Biology, University of California Berkeley, Berkeley, CA 94720, USA.

\section{Acknowledgements}

Dr. Mi Yeon Lee is thanked for help with plant cultivation, and Dr. Aymerick Eudes is thanked for providing data on plants engineered with the QsuB construct prior to publication. Leah Sloan is thanked for reviewing English grammar.

\section{Competing interests}

The authors declare that they have no competing interests except that HVS and DL have financial interest in Afingen Inc.

\section{Availability of data and materials}

The materials and datasets used and/or analyzed during the current study are available from the corresponding author on reasonable request. All sequences and plasmids developed under this project are further described in Additional files 3 and 4 and will be made publicly available through the ICE repository [31].

\section{Consent for publication}

Not applicable.

\section{Ethics approval and consent to participate}

Not applicable.

\section{Funding}

This work conducted by the Joint BioEnergy Institute was supported by the US Department of Energy, Office of Science, Office of Biological and Environmental Research under Contract No. DE-AC02-05CH11231 between Lawrence Berkeley National Laboratory and the US Department of Energy. CC was supported by Ecole Normale Supérieure de Cachan.

\section{Publisher's Note}

Springer Nature remains neutral with regard to jurisdictional claims in published maps and institutional affiliations.

Received: 31 October 2017 Accepted: 23 December 2017

Published online: 09 January 2018

\section{References}

1. Ruzicka K, Ursache R, Hejatko J, Helariutta Y. Xylem development-from the cradle to the grave. New Phytol. 2015;207(3):519-35.

2. Marriott PE, Gomez LD, McQueen-Mason SJ. Unlocking the potential of lignocellulosic biomass through plant science. New Phytol. 2016;209(4):1366-81.

3. Portillo M, Cabrera J, Lindsey K, Topping J, Andres MF, Emiliozzi M, Oliveros JC, Garcia-Casado G, Solano R, Koltai H, et al. Distinct and conserved transcriptomic changes during nematode-induced giant cell development in tomato compared with Arabidopsis: a functional role for gene repression. New Phytol. 2013;197(4):1276-90.

4. Scheller H, Ulvskov P. Hemicelluloses. Annu Rev Plant Biol. 2010;61(1):263-89.

5. Van Vleet JH, Jeffries TW. Yeast metabolic engineering for hemicellulosic ethanol production. Curr Opin Biotechnol. 2009;20(3):300-6.

6. Young $\mathrm{E}$, Lee $\mathrm{SM}$, Alper $\mathrm{H}$. Optimizing pentose utilization in yeast: the need for novel tools and approaches. Biotechnol Biofuels. 2010;3:24.

7. Searcy E, Flynn P, Ghafoori E, Kumar A. The relative cost of biomass energy transport. Appl Biochem Biotechnol. 2007;137-140(1-12):639-52. 
8. Loque D, Scheller HV, Pauly M. Engineering of plant cell walls for enhanced biofuel production. Curr Opin Plant Biol. 2015;25:151-61.

9. Franke R, Humphreys JM, Hemm MR, Denault JW, Ruegger MO, Cusumano JC, Chapple C. The Arabidopsis REF8 gene encodes the 3-hydroxylase of phenylpropanoid metabolism. Plant J. 2002;30(1):33-45.

10. Shadle G, Chen F, Srinivasa Reddy MS, Jackson L, Nakashima J, Dixon RA. Down-regulation of hydroxycinnamoyl CoA: shikimate hydroxycinnamoyl transferase in transgenic alfalfa affects lignification, development and forage quality. Phytochemistry. 2007;68(11):1521-9.

11. Voelker SL, Lachenbruch B, Meinzer FC, Jourdes M, Ki C, Patten AM, Davin LB, Lewis NG, Tuskan GA, Gunter L, et al. Antisense down-regulation of $4 C L$ expression alters lignification, tree growth, and saccharification potential of field-grown poplar. Plant Physiol. 2010;154(2):874-86.

12. Eudes A, Sathitsuksanoh N, Baidoo EE, George A, Liang Y, Yang F, Singh S, Keasling JD, Simmons BA, Loque D. Expression of a bacterial 3-dehydroshikimate dehydratase reduces lignin content and improves biomass saccharification efficiency. Plant Biotechnol J. 2015;13(9):1241-50.

13. Teramoto $H$, Inui M, Yukawa H. Regulation of expression of genes involved in quinate and shikimate utilization in Corynebacterium glutamicum. Appl Environ Microbiol. 2009;75(11):3461-8.

14. Eudes A, Pereira JH, Yogiswara S, Wang G, Teixeira Benites V, Baidoo EE, Lee TS, Adams PD, Keasling JD, Loque D. Exploiting the substrate promiscuity of hydroxycinnamoyl-CoA: shikimate hydroxycinnamoyl transferase to reduce lignin. Plant Cell Physiol. 2016;57(3):568-79.

15. Harholt J, Suttangkakul A, Scheller HV. Biosynthesis of pectin. Plant Physiol. 2010;153(2):384-95.

16. Gorshkova T, Mokshina N, Chernova T, Ibragimova N, Salnikov V, Mikshina P, Tryfona T, Banasiak A, Immerzeel P, Dupree P, et al. Aspen tension wood fibers contain beta- $(1->4)$-galactans and acidic arabinogalactans retained by cellulose microfibrils in gelatinous walls. Plant Physiol. 2015;169(3):2048-63.

17. Liwanag AJ, Ebert B, Verhertbruggen $Y$, Rennie EA, Rautengarten $C$, Oikawa A, Andersen MC, Clausen MH, Scheller HV. Pectin biosynthesis: GALS1 in Arabidopsis thaliana is a beta-1,4-galactan beta-1,4-galactosyltransferase. Plant Cell. 2012;24(12):5024-36.

18. Rosti J, Barton CJ, Albrecht S, Dupree P, Pauly M, Findlay K, Roberts K, Seifert GJ. UDP-glucose 4-epimerase isoforms UGE2 and UGE4 cooperate in providing UDP-galactose for cell wall biosynthesis and growth of Arabidopsis thaliana. Plant Cell. 2007;19(5):1565-79.

19. Gondolf VM, Stoppel R, Ebert B, Rautengarten C, Liwanag AJ, Loque $D$, Scheller HV. A gene stacking approach leads to engineered plants with highly increased galactan levels in Arabidopsis. BMC Plant Biol. 2014;14:344.

20. Rautengarten C, Ebert B, Moreno I, Temple H, Herter T, Link B, DonasCofre D, Moreno A, Saez-Aguayo S, Blanco F, et al. The Golgi localized bifunctional UDP-rhamnose/UDP-galactose transporter family of Arabidopsis. Proc Natl Acad Sci USA. 2014;111(31):11563-8.

21. Brown DM, Goubet F, Vicky WWA, Goodacre R, Stephens E, Dupree P, Turner SR. Comparison of five xylan synthesis mutants reveals new insight into the mechanisms of xylan synthesis. Plant J. 2007;52(6):1154-68.

22. Petersen PD, Lau J, Ebert B, Yang F, Verhertbruggen Y, Kim JS, Varanasi $P$, Suttangkakul A, Auer M, Loque D, et al. Engineering of plants with improved properties as biofuels feedstocks by vessel-specific complementation of xylan biosynthesis mutants. Biotechnol Biofuels. 2012;5(1):84.

23. Mitsuda N, Seki M, Shinozaki K, Ohme-Takagi M. The NAC transcription factors NST1 and NST2 of Arabidopsis regulate secondary wall thickenings and are required for anther dehiscence. Plant Cell. 2005;17(11):2993-3006

24. Yang F, Mitra P, Zhang L, Prak L, Verhertbruggen Y, Kim JS, Sun L, Zheng $\mathrm{K}$, Tang $\mathrm{K}$, Auer $\mathrm{M}$, et al. Engineering secondary cell wall deposition in plants. Plant Biotechnol J. 2013;11(3):325-35.
25. Shih PM, Vuu K, Mansoori N, Ayad L, Louie KB, Bowen BP, Northen TR, Loque D. A robust gene-stacking method utilizing yeast assembly for plant synthetic biology. Nat Commun. 2016;7:13215.

26. Taylor NG, Howells RM, Huttly AK, Vickers K, Turner SR. Interactions among three distinct CesA proteins essential for cellulose synthesis. Proc Natl Acad Sci USA. 2003;100(3):1450-5.

27. Persson S, Paredez A, Carroll A, Palsdottir H, Doblin M, Poindexter P, Khitrov N, Auer M, Somerville CR. Genetic evidence for three unique components in primary cell-wall cellulose synthase complexes in Arabidopsis. Proc Natl Acad Sci USA. 2007;104(39):15566-71.

28. Halpin C, Cooke SE, Barakate A, El Amrani A, Ryan MD. Self-processing 2A-polyproteins-a system for co-ordinate expression of multiple proteins in transgenic plants. Plant J. 1999;17(4):453-9.

29. Balsamo R, Boak M, Nagle K, Peethambaran B, Layton B. Leaf biomechanical properties in Arabidopsis thaliana polysaccharide mutants affect drought survival. J Biomech. 2015;48(15):4124-9.

30. Loque D, Liang Y. Generation of heritable chimeric plant traits. 2015. US patent application US20150218573.

31. Ham TS, Dmytriv Z, Plahar H, Chen J, Hillson NJ, Keasling JD. Design, implementation and practice of JBEI-ICE: an open source biological part registry platform and tools. Nucleic Acids Res. 2012;40(18):e141.

32. Shen WJ, Forde BG. Efficient transformation of Agrobacterium spp. by high voltage electroporation. Nucleic Acids Res. 1989;17(20):8385.

33. Clough SJ, Bent AF. Floral dip: a simplified method for Agrobacterium-mediated transformation of Arabidopsis thaliana. Plant J. 1998;16(6):735-43.

34. Czechowski T, Stitt M, Altmann T, Udvardi MK, Scheible WR. Genomewide identification and testing of superior reference genes for transcript normalization in Arabidopsis. Plant Physiol. 2005;139(1):5-17.

35. Schmittgen TD, Livak KJ. Analyzing real-time PCR data by the comparative C(T) method. Nat Protoc. 2008;3(6):1101-8.

36. Vandesompele J, De Preter K, Pattyn F, Poppe B, Van Roy N, De Paepe A, Speleman F. Accurate normalization of real-time quantitative RT-PCR data by geometric averaging of multiple internal control genes. Genome Biol. 2002;3(7):RESEARCH0034.

37. Jones L, Seymour GB, Knox JP. Localization of pectic galactan in tomato cell walls using a monoclonal antibody specific to (1[- > ]4)-[beta]-D-galactan. Plant Physiol. 1997;113(4):1405-12

38. Andersen MC, Boos I, Marcus SE, Kracun SK, Rydahl MG, Willats WG, Knox JP, Clausen MH. Characterization of the LM5 pectic galactan epitope with synthetic analogues of beta-1,4-d-galactotetraose. Carbohydr Res. 2016:436:36-40.

39. Schneider CA, Rasband WS, Eliceiri KW. NIH Image to ImageJ: 25 years of image analysis. Nat Methods. 2012;9(7):671-5.

40. Harholt J, Jensen JK, Sorensen SO, Orfila C, Pauly M, Scheller HV. ARABINAN DEFICIENT 1 is a putative arabinosyltransferase involved in biosynthesis of Pectic Arabinan in Arabidopsis. Plant Physiol. 2006;140(1):49-58.

41. Obro J, Harholt J, Scheller HV, Orfila C. Rhamnogalacturonan I in Solanum tuberosum tubers contains complex arabinogalactan structures. Phytochemistry. 2004;65(10):1429-38.

42. Yin L, Verhertbruggen $Y$, Oikawa A, Manisseri C, Knierim B, Prak L, Jensen JK, Knox JP, Auer M, Willats WG, et al. The cooperative activities of CSLD2, CSLD3, and CSLD5 are required for normal Arabidopsis development. Mol Plant. 2011;4(6):1024-37.

43. MacMillan CP, Mansfield SD, Stachurski ZH, Evans R, Southerton SG Fasciclin-like arabinogalactan proteins: specialization for stem biomechanics and cell wall architecture in Arabidopsis and Eucalyptus. Plant J. 2010;62(4):689-703.

44. Miller GL. Use of dinitrosalicylic acid reagent for determination of reducing sugar. Anal Chem. 1959;31(3):426-8. 